\title{
Ultrasound differentiation between benign versus malignant adnexal masses in pregnant patients
}

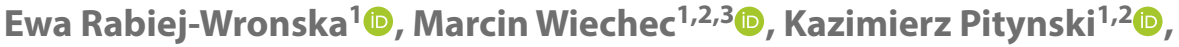 \\ Ewa Wiercinska ${ }^{4}$, Agnieszka Kotlarz ${ }^{1,2}$ (B) \\ 'Gynaecology and Oncology Clinical Department, University Hospital, Cracow, Poland \\ ${ }^{2}$ Chair of Gynecology and Obstetrics, Jagiellonian University Medical College, Cracow, Poland \\ ${ }^{3}$ Centre of Postgraduate Medical Education, Warsaw, Poland \\ ${ }^{4}$ District Sanitary Epidemiologic Station, Wieliczka, Poland
}

\begin{abstract}
Objectives: The aim of this study was to assess the performance of the International Ovarian Tumor Analysis (IOTA) group ultrasound Simple Rules method in differentiating between benign and malignant ovarian tumors in pregnant patients.

Material and methods: A prospective observational study that involved pregnant patients referred to our center due to suspicions of ovarian masses between January 2015 and December 2017 was performed. The Simple Rules performance was evaluated against the histopathological results. Each of the 10 sonographic Simple Rules were computed by logistic regression to demonstrate their odds ratios in predicting malignancy.

Results: Ultrasound were conducted in 153 subjects, and 61 of those patients underwent surgery. By assigning masses presenting inconclusive picture as probably malignant, the Simple Rules method showed sensitivity of $91.67 \%$ and specificity of $69.39 \%$. After exclusion of masses with inconclusive findings, the method showed sensitivity of $87.5 \%$ and specificity of $94.44 \%$. The Simple Rules risk estimation method for the $1 \%$ risk cutoff showed sensitivity of $100 \%$ and specificity of $51.02 \%$. For the $3 \%$ cutoff, sensitivity was $91.67 \%$ and specificity was $53.06 \%$. And for $30 \%$ cutoff, sensitivity was $91.67 \%$ and specificity $73.47 \%$. The logistic regression model showed that the M-rules increased the risk of malignancy while the B-rules decreased the risk.

Conclusions: Most ovarian masses in pregnant patients may be correctly categorized as benign or malignant using Simple Rules. This protocol may facilitate the management of pregnant patients presenting with adnexal masses.
\end{abstract}

Key words: pregnancy; adnexal diseases; ovarian neoplasms; ultrasonography

Ginekologia Polska 2022; 93, 8: 643-649

\section{INTRODUCTION}

The prevalence of adnexal masses during pregnancy is estimated to be $0.2-2 \%$ [1]. The majority of these masses are benign and include functional corpus luteum cysts, dermoid cysts, cystadenomas and endometriomas as the most common findings [2, 3]. Heterotopic pregnancy, tubo-ovarian abscesses, ovarian hyperstimulation and adnexal torsion should also be taken into consideration. Adnexal masses are identified during pregnancy with increasing frequency at the time of viability scans [4]. Most patients are asymptomatic, and the ovarian tumors are discovered incidentally [5].

Up to $70 \%$ of all ovarian cysts diagnosed in the first trimester resolve spontaneously later in gestation [6]. It is estimated that approximately $0.8-13 \%$ of all ovarian masses detected in pregnant women are malignant (ovarian cancer or metastatic tumors) $[7,8]$. One potential reason for this incidence is increasing maternal age [9]. Primary malignant tumors seem to have no impact on neonatal outcomes, metastases to ovaries however may lead to complications of the newborn period [10].

Due to the limited application of biomarkers for ovarian malignancy screening, according to the International Ovarian Tumor Analysis (IOTA) group, sonography remains the first-line imaging modality used for this purpose, including during pregnancy $[11,12]$. Several screening protocols have been described in the literature [13-16]. However, no

\footnotetext{
Corresponding author:

Ewa Rabiej-Wronska

Gynaecology and Oncology Clinical Department, University Hospital, 2 Jakubowskiego St, 30-688 Cracow, Poland

e-mail: ewa.rabiej@mailmix.pl
} 
Table 1. The simple rules

\begin{tabular}{|l|l|l|l|}
\hline \multicolumn{2}{|l|}{ M-rules for predicting a malignant tumor } & B-rules for predicting a benign tumor \\
\hline M1 & Irregular solid tumor & B1 & Unilocular cyst \\
\hline M2 & Presence of ascites & B2 & $\begin{array}{l}\text { Presence of solid components, where the largest solid } \\
\text { component has a diameter }<7 \mathrm{~mm}\end{array}$ \\
\hline M3 & At least 4 papillary structures & B3 & Presence of acoustic shadows \\
\hline M4 & $\begin{array}{l}\text { Irregular multilocular-solid tumor with largest } \\
\text { diameter } \geq 100 \mathrm{~mm}\end{array}$ & B4 & Smooth multilocular tumor with largest diameter $<100 \mathrm{~mm}$ \\
\hline M5 & Strong blood flow (color score 4) & B5 & No blood flow \\
\hline
\end{tabular}

policy regarding ovarian cancer screening during pregnancy has been proposed so far [5].

Indications for surgery to remove an adnexal mass during pregnancy are suspicion of malignancy, fast growth and large size of the tumor, or emergencies related to the adnexal mass, e.g., rupture or torsion [17]. If surgery is indicated, the optimal time of it to be performed is during the second trimester $[18,19]$

\section{Objectives}

Taking the above information into account, we planned an observational study in which we decided to determine if the sonographic criteria for malignancy described in the literature for nonpregnant subjects are also applicable for pregnant women.

\section{MATERIAL AND METHODS}

We performed a prospective observational study that involved pregnant subjects referred to our oncology center because of ovarian masses. Data were collected between January 2015 and December 2017. The exclusion criteria were refusal to participate and nonpregnant at the time of qualification for the study. All subjects underwent combined pelvic transvaginal and transabdominal sonograms performed using Voluson E6 ultrasound systems equipped with 5-9 MHz transvaginal and 2-5 MHz transabdominal hybrid transducers (GE Healthcare, Zipf, Austria). If an ovarian mass was confirmed, then it was evaluated by grayscale and Power Doppler mapping (with PRF at the level of $0.6 \mathrm{kHz}$ ). To minimalize an inter observer errors number of observers was limited to two certified by IOTA examiners.

All masses were reported using the IOTA group terminology [20]. Based on the identified findings, the Simple Rules protocol was applied [15]. AsTimmerman et al., proposed, sonographic features of a tumor can be divided in two groups: predicting malignant (M-rules) and benign (B-rules) tumors (Tab. 1). If one or more M-rules and no B-rules apply, a mass is classified as malignant. Conversely, when one or more B-rules and no M-rules is present, mass is classified as benign. The result is called inconclusive when there are no $B$ and $M$ findings or pictures from both categories are identified [15].
On the basis of $\mathrm{M}$ - and B-rules it is also possible to estimate the risk of malignancy (given in percentages), which allows to classify all adnexal masses (without inconclusive results) [21]

Clinical records of all subjects were reviewed and compared based on the following parameters: age, gravidity, gestational age at the time of diagnosis, and Ca- 125 serum levels. Serum Ca-125 was measured with electrochemiluminescence immunoassay (ECLIA) method using Cobas 8000 analyzer (Roche Diagnostics, Mannheim, Germany).

All scan reports were reviewed by the oncology team, and subjects were counseled regarding the scan findings and underwent routine evaluation according to the local policy. The examiners were not involved in decision making process regarding patients' therapies. Second ultrasound scans were performed at this level. Every patient underwent follow-up ultrasound scans every 2-4 weeks till surgery. All cases qualified by the oncology team for surgical treatment were reviewed in terms of histopathology evaluation, including cases operated on after conclusion of the pregnancy. Subjects diagnosed with malignancy were included in Group $M$, and those with benign masses were included in Group B. For the purpose of the study, lesions indicating borderline characteristics on histopathology were classified as malignant (Group M). The follow-up ultrasound scan was scheduled between 6 and 20 weeks postpartum. The local ethics committee approval was acquired, and a written informed consent was obtained from all patients.

\section{Statistical analysis}

Normality of the continuous variable distribution was validated with the Kolmogorov-Smirnov test. The $x^{2}$ test was used to show differences. None of the continuous variables presented a Gaussian distribution. Nonparametric tests were conducted. Two groups of independent variables were compared in this case using the Mann-Whitney U-test.

All 10 of the sonographic features (M-rules and B-rules) were computed by logistic regression with one categorical independent variable to demonstrate their odds ratios in predicting malignancy.

The Simple Rules performance was evaluated against the histopathological results, which was the reference stand- 


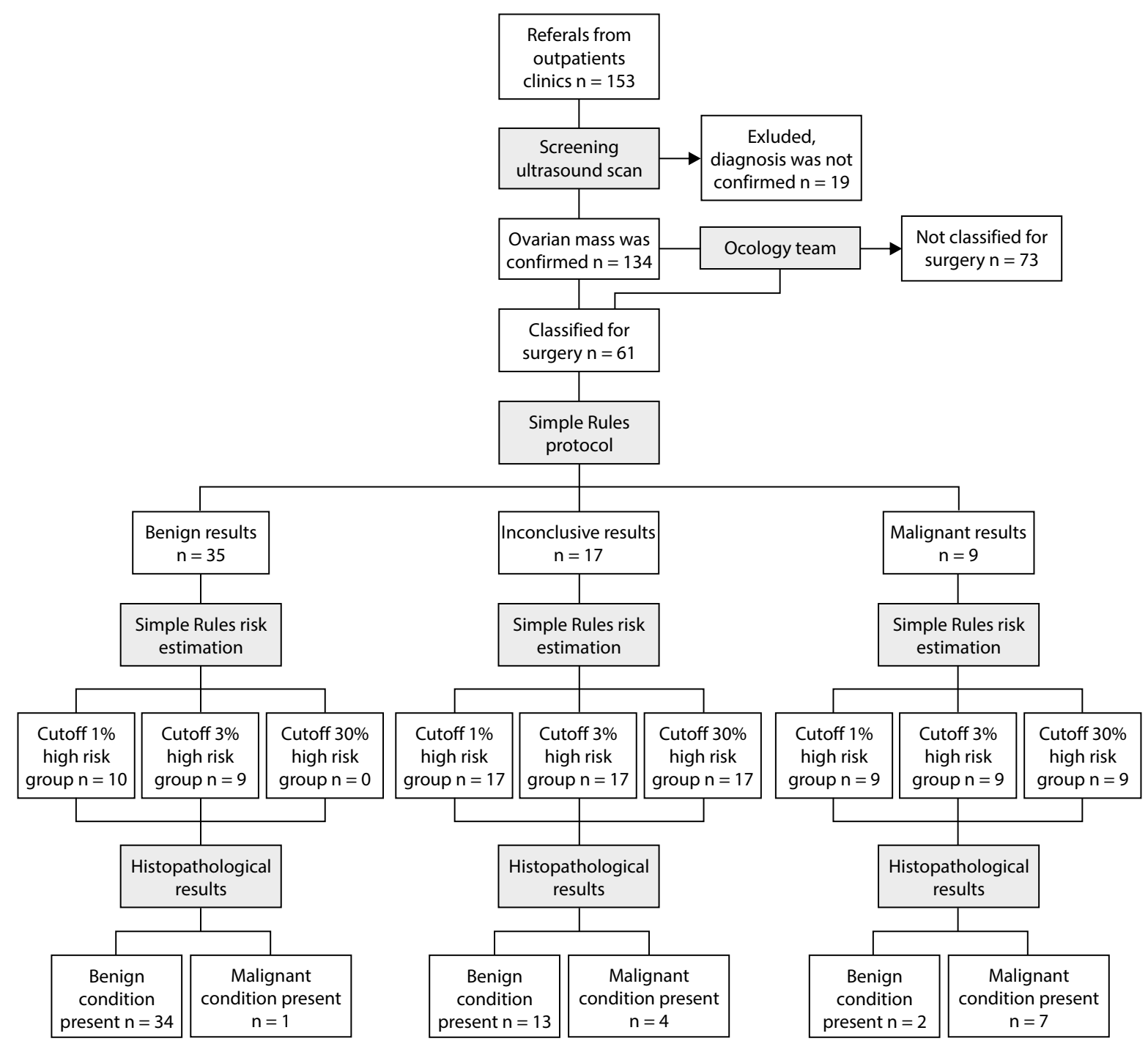

Figure 1. Study population breakdown diagram

ard in this case. Sensitivity, specificity, positive predictive value (PPV), negative predictive value (NPV), positive likelihood ratio (LR+), negative likelihood ratio (LR-) and diagnostic accuracy were calculated for the Simple Rules for all patients who underwent surgery.

Five calculation strategies were applied. First method, by assigning masses presenting inconclusive picture (no B and $M$ features or features from both categories) as probably malignant (Calculation 1), second, by excluding masses with inconclusive findings (Calculation 2), third, fourth and fifth, by calculating risk of malignancy with Simple Rules risk calculator at 1\% (Calculation 3), 3\% (Calculation 4) and 30\% (Calculation 5) cutoffs [21]. Statistical analysis was carried out using the Statistica 13 system (StatSoft, (racow, Poland). The values of $p<0.05$ were considered as statistically significant.

\section{RESULTS \\ Study population}

A total of 153 subjects diagnosed with an ovarian mass associated with pregnancy were referred to our oncology center. In 19 patients, ovarian masses were excluded by sonography at the time of qualification. The remaining 134 subjects were counseled by the oncology team. The diagnosis of ovarian mass was confirmed by sonography in all of 134 patients. Additionally, 73 subjects were not qualified for surgery, and thus, the remaining 61 patients constituted the study population and underwent surgery due to ovarian masses with histopathology evaluation. The details are shown in Figure 1. The surgery was performed during pregnancy in 35 subjects ( 4 patients with malignant, 5 with borderline and 26 with benign tumors) and the mean time between the suspicion on sonography and the first surgery was 
Table 2. The number of surgeries performed on the basis of particular indications

\begin{tabular}{|l|c|c|c|c|c|c|} 
& \multicolumn{3}{|c|}{$\begin{array}{c}\text { Group B } \\
\text { tumor removed: }\end{array}$} & \multicolumn{3}{c|}{$\begin{array}{c}\text { Group M } \\
\text { tumor removed: }\end{array}$} \\
\hline & $\begin{array}{c}\text { During } \\
\text { pregnancy } \\
\mathbf{n = 2 6}\end{array}$ & $\begin{array}{c}\text { During } \\
\text { cesarean } \\
\text { section } \mathbf{n = 1 2}\end{array}$ & $\begin{array}{c}\text { After } \\
\text { pregnancy } \\
\mathbf{n = 1 1}\end{array}$ & $\begin{array}{c}\text { During } \\
\text { pregnancy } \\
\mathbf{n = 1 0}\end{array}$ & $\begin{array}{c}\text { During } \\
\text { cesarean } \\
\text { section } \mathbf{n = 2}\end{array}$ & $\begin{array}{c}\text { After } \\
\text { pregnancy } \\
\mathbf{n = 0}\end{array}$ \\
\hline $\begin{array}{l}\text { Suspicious of malignancy (M features } \\
\text { or fast growth of the tumor) }\end{array}$ & 8 & 2 & 1 & 10 & 1 & 0 \\
\hline Torsion or rupture & 4 & 1 & 1 & 0 & 0 & 0 \\
\hline Chronic abdominal pain & 6 & 0 & 2 & 0 & 0 & 0 \\
\hline Other symptoms of lesions & 8 & 0 & 0 & 0 & 0 & 0 \\
\hline $\begin{array}{l}\text { Tumor as an obstacle for vaginal } \\
\text { delivery }\end{array}$ & 0 & 3 & 0 & 0 & 1 & 0 \\
\hline $\begin{array}{l}\text { Obstetric indications for cesarean } \\
\text { section }\end{array}$ & 0 & 6 & 0 & 0 & 0 & 0 \\
\hline $\begin{array}{l}\text { Tumor persistence after course of } \\
\text { pregnancy }\end{array}$ & 0 & 0 & 7 & 0 & 0 & 0 \\
\hline
\end{tabular}

If patient has more than one surgery, only first one is included in the table

Table 3. Basic statistics of major study population parameters: maternal age, gestational age, and Ca-125

\begin{tabular}{|l|c|c|c|c|c|c|c|}
\hline & \multicolumn{3}{|c|}{ Benign } & \multicolumn{3}{|c|}{ Malignant } \\
\hline & Mean & Median & SD & Mean & Median & SD & Statistical significance \\
\hline Maternal age & 28.94 & 28.00 & 5.48 & 29.83 & 28.50 & 6.09 & 0.68 \\
\hline Gestational age & 16.37 & 15.00 & 7.66 & 16.04 & 17.79 & 4.69 & 0.82 \\
\hline Ca-125 level [U/mL] & 41.37 & 27.56 & 38.11 & 93.49 & 33.40 & 159.12 & 0.90 \\
\hline
\end{tabular}

2.5 weeks (range $0-8$ weeks). In 14 subjects, surgery was performed at the time of cesarean section (one case of mucinous carcinoma, one case of seromucionous borderline tumor and 12 benign cases), and the mean time from the suspicious sonogram findings to the surgery was 22 weeks (range 8-31 weeks). In 11 subjects, surgery was performed after the course of pregnancy (only benign tumors) and the mean time from the first scan to the surgery was 23.5 weeks (range 11-36 weeks). Patient with Burkitt lymphoma was diagnosed with ovarian mass at 19 weeks of gestation, and her first surgery was performed at 26 weeks and consecutive removal of recurrences during cesarean section performed at 31 weeks.

Indications for surgeries are shown in Table 2. In our study population there were six cases of emergency surgical interventions due to torsion or rupture of the mass. In all these six subjects Simple Rules suggested benign character of the tumor, and it was confirmed in histopathology.

The median maternal age was 30 years (range: $16-44$, SD 5.1 years). The median maternal age for patients in Group $M$ was 28.5 years (range: 19-41, SD 6.09 years) and 28 years (range: 18-44, SD 5.48 years) in Group B (no statistical significance $p=0.68$ ). Gestational age at diagnosis and $\mathrm{Ca}-125$ data are shown in Table 3. There were no statistical differences between the two groups regarding maternal age, gestational age at the time of sonography or Ca-125 levels (Tab. 3).

In Group B there were 34 patients (69.4\%) in their first pregnancy, 12 (24.5\%) in their second, 2 (4.1\%) in their third, and $1(2 \%)$ in her fifth. In Group $M$ there were 7 patients $(58.3 \%)$ in their first, 3 (25\%) in their second and 2 (16.7\%) in their third pregnancy.

\section{Histopathology}

The malignancy rate was $7.84 \%$ (12/153). Among the studied subjects, a benign teratoma was the most common finding. Detailed histopathology results are presented in Table 4.

\section{Ultrasound findings}

Among the benign lesions there were 21 masses (42.9\%) described as unilocular, 11 (22.4\%) as multilocular, 8 (16.3\%) as unilocular-solid, $6(12.2 \%)$ as multilocular-solid and $3(6.1 \%)$ as solid. In Group M, 2 masses (16.7\%) were unilocular, 3 (25\%) unilocular-solid, 3 (25\%) multilocular-solid and 4 (33.3\%) solid. There were no multilocular lesions in Group M. The dominant sonographic features in Group B were anechoic in 12 masses (24.5\%), low level of echogenicity in $5(10.2 \%)$, ground glass in $6(12.2 \%)$, hemorrhagic in 


\begin{tabular}{|c|c|}
\hline Prevalence of specific pathologies & \\
\hline Tumor type & n (\%) \\
\hline All benign & 52 \\
\hline Benign teratoma & $19(29.69)$ \\
\hline Endometrioma & $7(10.94)$ \\
\hline Simple cyst & $7(10.94)$ \\
\hline Functional cyst & $3(4.8)$ \\
\hline Parasalpingeal cyst & $2(3.13)$ \\
\hline Mucinous cystadenoma & $8(12.5)$ \\
\hline Fibroma & $2(3.13)$ \\
\hline Fibrothecoma & $2(3.13)$ \\
\hline Cystadenofibroma & $2(3.13)$ \\
\hline All malignant & 12 \\
\hline Serous borderline tumor & $3(4.69)$ \\
\hline Mucinous borderline tumor & $1(1.56)$ \\
\hline Seromucinous borderline tumor & $2(3.13)$ \\
\hline Yolk sac tumor & $2(3.13)$ \\
\hline Serous carcinoma & $1(1.56)$ \\
\hline Mucinous carcinoma & $1(1.56)$ \\
\hline Burkitt lymphoma & $1(1.56)$ \\
\hline Secondary metastatic cancer & $1(1.56)$ \\
\hline
\end{tabular}

There were three tumors of mixed histology: yolk sac tumor together with mucinous cystadenoma, yolk sac tumor together with benign teratoma, and endometrioma together with a simple cyst. These tumors are shown as separate diagnoses

$4(8.2 \%)$ and as mixed in 22 (44.9\%). In Group M there was 1 mass (8.3\%) described as anechoic, 1 as low level (8.3\%), 1 as ground glass (8.3\%), 2 as hemorrhagic (16.7\%) and 7 as mixed (58.3\%). Color score 1 was given for 37 (75.5\%) benign tumors, color score 2 for 7 (14.3\%), 3 for 5 (10.2\%) and 4 for none of them. One (8.3\%) malignant tumor had color a score of 1 , four (33.3\%) had a color score of 2, six (50\%) a color score 3 and only one (8.3\%) a color score of 4 . Differences in type, dominant sonographic features and blood flow between benign and malignant masses were not tested for statistical significance because of the small number of tumors in each particular group.

All 10 of the sonographic features (M-rules and B-rules) were computed by logistic regression with one categorical independent variable. Odds ratios of predicting malignancy are presented in Table 5.

The results were statistically significant for rules $\mathrm{M} 1$ - irregular solid tumor (OR 7.83), M2 - ascites (OR 16), M3 - at least four papillary structures (OR 34.29), M4 — irregular multilocular-solid tumor with largest diameter $\geq 100 \mathrm{~mm}$ (OR 5.11), B1 - unilocular cyst (OR 0.1), B5 — no blood flow (OR 0.04). M5 - very strong blood flow was present only in two cases, but all of them were malignant (2/2); B2 - pres-
Table 5. Odds ratios (ORs) for the $M$ and $B$ features based on the logistic regression model

\begin{tabular}{|l|c|c|c|c|}
\hline & p & OR & \multicolumn{2}{|c|}{$95 \%$ Cl for OR } \\
\hline & & & Lower limit & Upper limit \\
\hline M1* & 0.036 & 7.833 & 1.141 & 53.757 \\
\hline M2* & 0.022 & 16 & 1.49 & 171.59 \\
\hline M3* & 0.002 & 34.286 & 3.476 & 338.16 \\
\hline M4* & 0.068 & 5.111 & 0.886 & 29.486 \\
\hline M5* & 0.999 & 7915826838 & 0 & \\
\hline B1 $^{*}$ & 0.03 & 0.095 & 0.011 & 0.791 \\
\hline B2 $^{*}$ & 0.999 & 0 & 0 & \\
\hline B3 $^{*}$ & 0.999 & 0 & 0 & \\
\hline B4 $^{*}$ & 0.999 & 0 & 0 & \\
\hline B5 $^{*}$ & 0.003 & 0.04 & 0.005 & 0.339 \\
\hline
\end{tabular}

* - definition in the Table 1

ence of solid components, where the largest has a diameter $<7 \mathrm{~mm}$, was present in three tumors, all of them benign (3/3); B3 - presence of acoustic shadows was present in five tumors, all of them benign (5/5); and B4 - smooth multilocular tumor with a largest diameter $<100 \mathrm{~mm}$ was present in three tumors, all of them benign (3/3).

\section{Key findings}

Screening performance of all proposed by us Calculation models is shown in Table 6.

\section{Follow-up}

A total of 86 (63\%) patients attended a postpartum follow-up scan. In the group of patients who did not undergo surgery, ultrasounds showed normal ovaries in 37 subjects and ovarian cyst in 11 subjects. All of 11 ovarian masses indicated benign character (10 endometriomas and one simple cyst). Among patients who underwent surgery normal ovaries were found in 37 subjects ( 2 of those patients were in consecutive pregnancy). In one subject an ovarian cyst was found. It was a dermoid cyst in a patient who underwent cystectomy due to dermoid cyst in contralateral ovary.

\section{DISCUSSION}

Simple Rules performance has been already evaluated in multicenter studies conducted by the IOTA group on a population of nonpregnant women [22]. To the best of our knowledge, our study is one of the first performed on a population of pregnant women [10]. The results of this study confirmed that Simple Rules reliably discriminated between benign and malignant adnexal masses detected in pregnant subjects.

One of the strengths of the study is the use of the same standardized lesion qualification as previously used in 
Table 6. Screening performance

\begin{tabular}{|c|c|c|c|c|c|}
\hline & $\begin{array}{l}\text { Calculation model } 1 \\
\text { (inconclusive } \\
\text { results as probably } \\
\text { malignant) }\end{array}$ & $\begin{array}{l}\text { Calculation model } 2 \\
\text { (exclusion of } \\
\text { inconclusive results) }\end{array}$ & $\begin{array}{c}\text { Calculation model } 3 \\
1 \% \text { risk cutoff }\end{array}$ & $\begin{array}{c}\text { Calculation model } 4 \\
3 \% \text { risk cutoff }\end{array}$ & $\begin{array}{c}\text { Calculation model } 5 \\
30 \% \text { risk cutoff }\end{array}$ \\
\hline Sensitivity & $\begin{array}{c}91.67 \% \\
\text { Cl } 61.52-99.79\end{array}$ & $\begin{array}{c}87.5 \% \\
\text { Cl } 47.35-99.68\end{array}$ & $\begin{array}{c}100 \% \\
\text { Cl } 73.54-100\end{array}$ & $\begin{array}{c}91.67 \% \\
\text { Cl } 61.52-99.79\end{array}$ & $\begin{array}{c}91.67 \% \\
\text { CI } 61.52-99.79\end{array}$ \\
\hline Specificity & $\begin{array}{c}69.39 \% \\
\text { CI } 54.58-81.75\end{array}$ & $\begin{array}{c}94.44 \% \\
\text { CI } 81.34-99.32\end{array}$ & $\begin{array}{c}51.02 \% \\
\text { CI } 36.34-65.58\end{array}$ & $\begin{array}{c}53.06 \% \\
\text { CI } 38.27-67.47\end{array}$ & $\begin{array}{c}73.47 \% \\
\text { CI } 58.92-85.05\end{array}$ \\
\hline $\begin{array}{l}\text { Diagnostic } \\
\text { accuracy }\end{array}$ & $\begin{array}{c}73.77 \% \\
\text { Cl } 60.93-84.20\end{array}$ & $\begin{array}{c}93.18 \% \\
\text { Cl } 81.77-97.65\end{array}$ & $\begin{array}{c}60.66 \% \\
\text { Cl } 47.31-72.93\end{array}$ & $\begin{array}{c}60.66 \% \\
\text { Cl } 47.31-72.93\end{array}$ & $\begin{array}{c}77.05 \% \\
\text { Cl } 47.31-72.93\end{array}$ \\
\hline PPV & $\begin{array}{c}42.31 \% \\
\text { Cl } 31.76-53.61\end{array}$ & $\begin{array}{c}77.78 \% \\
\text { Cl } 47.02-93.24\end{array}$ & $\begin{array}{c}33.33 \% \\
\text { Cl } 27.31-39.95\end{array}$ & $\begin{array}{c}32.35 \% \\
\text { CI } 25.34-40.26\end{array}$ & $\begin{array}{c}45.83 \% \\
\mathrm{Cl} 34.00-58.16\end{array}$ \\
\hline NPV & $\begin{array}{c}97.14 \% \\
83.76-99.56\end{array}$ & $\begin{array}{c}97.14 \% \\
\text { Cl } 84.44-99.53\end{array}$ & $100 \%$ & $\begin{array}{c}96.30 \% \\
\text { Cl } 79.63-99.43\end{array}$ & $\begin{array}{c}97.3 \% \\
\text { Cl } 84.55-99.58\end{array}$ \\
\hline LR+ & $\begin{array}{c}2.99 \\
\text { Cl } 1.90-4.72\end{array}$ & $\begin{array}{c}15.75 \\
\text { Cl } 3.99-62.11\end{array}$ & $\begin{array}{c}2.04 \\
\text { Cl } 1.53-2.72\end{array}$ & $\begin{array}{c}1.95 \\
\text { Cl } 1.39-2.75\end{array}$ & $\begin{array}{c}3.46 \\
\text { Cl } 2.10-5.67\end{array}$ \\
\hline LR- & $\begin{array}{c}0.12 \\
\text { Cl } 0.02-0.79\end{array}$ & $\begin{array}{c}0.13 \\
\mathrm{Cl} 0.02-0.83\end{array}$ & 0 & $\begin{array}{c}0.16 \\
\text { Cl } 0.02-1.04\end{array}$ & $\begin{array}{c}0.11 \\
\mathrm{Cl} 0.02-0.75\end{array}$ \\
\hline
\end{tabular}

nonpregnant subjects. The results may therefore be compared. The Simple Rules performance in nonpregnant showed a sensitivity of $95 \%$ and specificity of $78 \%$ if inconclusive results were classified as probably malignant and sensitivity of $92 \%$ and a specificity of $96 \%$ after exclusion masses with inconclusive result [22]. Simple Rules risk estimation with $1 \%$ risk cutoff showed sensitivity of $99.7 \%$ and a specificity of $33.7 \%$, and with $30 \%$ cutoff, sensitivity of $89.0 \%$ and specificity of $84.7 \%$ [21].

These results are in line with our findings in pregnant women. Another similarity is that the same as in nonpregnant population, the presence of $M$-features increases the risk of malignancy, whereas the presence of B-features decreases this risk [15].

However, several observations were dissimilar. In pregnant subjects, the M3 feature (at least 4 papillary structures) was the most predictive for malignancy, contrary to the IOTA group studies on nonpregnant subjects where, the most predictive feature was M2 (ascites). Nevertheless, both studies suggested that M4 (irregular multilocular-solid tumor with a largest diameter larger than $100 \mathrm{~mm}$ ) is the least predictive one. Moreover, the B5 feature (no vascular signals within the lesion) was the most predictive for the benign character, while B1 (unilocular cyst) was the least predictive. In the IOTA group studies the B1 feature was the most predictive of a benign character and B3 (presence of acoustic shadows) was the least predictive [21]. These differences in tumor characteristics may be probably explained by the younger age of our pregnant subjects. The median maternal age for patients in our Group M was 28.5 years (range: 19-41 years) and 28 years (range: 18-44 years) in Group B. In the IOTA studies the group of patients consisted of both pre- and postmenopausal women and their mean age was
57 years (range: 47-66 years) for patients with a malignant tumor and 42 years (range: 32-54 years) for patients with a benign condition [21]. We would also argue that the pregnancy itself likely influence sonographic appearance of the tumor by inducing hormonal dependent changes in tissues and accelerating the blood flow in pelvic vessels. Moreover, the visualization of ovaries may by difficult due to volume of pregnant uterus. These hypotheses however require detailed verification.

Among the strengths of our study are also its prospective protocol and the inclusion of IOTA certified examiners. Moreover, only patients who underwent surgery with detailed histopathology assessment were included.

Simple Rules in their original version is applicable in $72 \%$ of adnexal masses in pregnant subjects. This result is in line with the figure of $76 \%$ observed in nonpregnant women by other researchers [15]. For inconclusive results proposed approach is to qualify the lesion as potentially malignant or to use Simple Rules risk calculation [21]. However, definition of applicable cutoffs for pregnant subjects is necessary to enhance screening potential of risk of malignancy calculation models. So far, there is a general agreement that for masses that cannot be simply classified, scan performed by an expert sonologist should be offered [21, 22].

Limitations of the study are the fact that the data comes from a single center and the final database consisted of 61 subjects only. The above-mentioned facts indicate main future research direction. Larger series are necessary to confirm our findings in pregnant subjects.

\section{CONCLUSIONS}

This study suggests that majority of ovarian masses in pregnant patient are correctly categorized as benign 
or malignant using Simple Rules. If there is no tool dedicated to ovarian cancer screening in pregnant patients, Simple Rules may fill this gap. There is no question that for both pregnant and nonpregnant subjects, sonography performed by an expert is the method with the highest sensitivity for discriminating between malignant and benign adnexal lesions [21, 22]. However, the Simple Rules method may help less experienced examiners to achieve reliable qualification of adnexal masses in pregnant women. The common usage of Simple Rules would probably reduce the number of patients that need to be referred for expert ultrasound scanning. The development of the optimal tool dedicated to ovarian mass assessment in pregnant patients will require further prospective multi-center studies and the involvement of larger cohorts of patients.

\section{Acknowledgements}

The work was supported by the Department of Gynecology and Oncology, University Hospital in Cracow, Poland.

\section{Conflict of interest}

The authors state that there are no conflicts of interest to disclose.

\section{REFERENCES}

1. de Haan J, Verheecke M, Amant F. Management of ovarian cysts and cancer in pregnancy. Facts Views Vis Obgyn. 2015; 7(1): 25-31, indexed in Pubmed: 25897369.

2. Fruscio R, de Haan J, Van Calsteren $\mathrm{K}$, et al. Ovarian cancer in pregnancy. Best Pract Res Clin Obstet Gynaecol. 2017; 41: 108-117, doi: 10.1016/j. bpobgyn.2016.09.013, indexed in Pubmed: 28029502.

3. Leiserowitz GS. Managing ovarian masses during pregnancy. Obstet Gynecol Surv. 2006;61(7):463-470, doi:10.1097/01.ogx.0000224614.51356. b7, indexed in Pubmed: 16787549.

4. Leiserowitz GS, Xing G, Cress R, et al. Adnexal masses in pregnancy: how often are they malignant? Gynecol Oncol. 2006; 101(2): 315-321, doi: 10.1016/j.ygyno.2005.10.022, indexed in Pubmed: 16310839.

5. Hakoun AM, AbouAl-Shaar I, Zaza KJ, et al. Adnexal masses in pregnancy: An updated review. Avicenna J Med. 2017; 7(4): 153-157, doi: 10.4103/ajm.AJM 22 17, indexed in Pubmed: 29119081.

6. Bernhard L, Klebba PK, Gray D, et al. Predictors of persistence of adnexal masses in pregnancy. Obstetrics \& Gynecology. 1999; 93(4): 585-589, doi: 10.1016/s0029-7844(98)00490-6.

7. Mukhopadhyay A, Shinde A, Naik R. Ovarian cysts and cancer in pregnancy. Best Pract Res Clin Obstet Gynaecol. 2016; 33: 58-72, doi: 10.1016/j.bpobgyn.2015.10.015, indexed in Pubmed: 26707193.

8. Webb KE, Sakhel K, Chauhan SP, et al. Adnexal mass during pregnancy: a review. Am J Perinatol. 2015; 32(11): 1010-1016, doi: 10.1055/s-00351549216, indexed in Pubmed: 26007316.

9. Palmer J, Vatish M, Tidy J. Epithelial ovarian cancer in pregnancy: a review of the literature. BJOG. 2009; 116(4): 480-491, doi: 10.1111/j.1471-0528 .2008.02089.x, indexed in Pubmed: 19250360.
10. Moro F, Mascilini F, Pasciuto T, et al. Ultrasound features and clinical outcome of patients with malignant ovarian masses diagnosed during pregnancy: experience of a gynecological oncology ultrasound center Int J Gynecol Cancer. 2019; 29(7): 1182-1194, doi: 10.1136/ijgc-2019000373, indexed in Pubmed: 31326950.

11. Van Calster B, Timmerman D, Bourne $T$, et al. Discrimination between benign and malignant adnexal masses by specialist ultrasound examination versus serum CA-125. J Natl Cancer Inst. 2007; 99(22): 1706-1714, doi: 10.1093/jnci/djm199, indexed in Pubmed: 18000221.

12. Valentin $L$, Jurkovic $D$, Van Calster $B$, et al. Adding a single $C A$ 125 measurement to ultrasound imaging performed by an experienced examiner does not improve preoperative discrimination between benign and malignant adnexal masses. Ultrasound Obstet Gynecol. 2009; 34(3): 345-354, doi: 10.1002/uog.6415, indexed in Pubmed: 19585547.

13. Jacobs I, Oram D, Fairbanks J, et al. A risk of malignancy index incorporating CA 125, ultrasound and menopausal status for the accurate preoperative diagnosis of ovarian cancer. Br J Obstet Gynaecol. 1990; 97(10): 922-929, doi: 10.1111/j.1471-0528.1990.tb02448.x, indexed in Pubmed: 2223684.

14. Timmerman D, Testa AC, Bourne T, et al. International Ovarian Tumor Analysis Group. Logistic regression model to distinguish between the benign and malignant adnexal mass before surgery: a multicenter study by the International Ovarian Tumor Analysis Group. J Clin Oncol. 2005; 23(34): 8794-8801, doi: 10.1200/JCO.2005.01.7632, indexed in Pubmed: 16314639.

15. Timmerman D, Testa AC, Bourne $T$, et al. Simple ultrasound-based rules for the diagnosis of ovarian cancer. Ultrasound Obstet Gynecol. 2008; 31(6): 681-690, doi: 10.1002/uog.5365, indexed in Pubmed: 18504770.

16. Van Calster B, Van Hoorde K, Valentin L, et al. International Ovarian Tumour Analysis Group. Evaluating the risk of ovarian cancer before surgery using the ADNEX model to differentiate between benign, borderline, early and advanced stage invasive, and secondary metastatic tumours: prospective multicentre diagnostic study. BMJ. 2014; 349: g5920, doi: 10.1136/bmj.g5920, indexed in Pubmed: 25320247.

17. Zanetta G, Mariani E, Lissoni A, et al. A prospective study of the role of ultrasound in the management of adnexal masses in pregnancy. BJOG. 2003; 110(6): 578-583, doi: 10.1046/j.1471-0528.2003.02940.x, indexed in Pubmed: 12798475.

18. Whitecar $P$, Turner $S$, Higby K. Adnexal masses in pregnancy: A review of 130 cases undergoing surgical management. Am J Obstet Gynecol. 1999; 181(1): 19-24, doi: 10.1016/s0002-9378(99)70429-1, indexed in Pubmed: 10411786

19. D'Ambrosio V, Brunelli R, Musacchio L, et al. Adnexal masses in pregnancy: an updated review on diagnosis and treatment. Tumori. 2021. 107(1): 12-16, doi: 10.1177/0300891620909144, indexed in Pubmed: 32180534.

20. Timmerman D, Valentin L, Bourne $\mathrm{TH}$, et al. International Ovarian Tumor Analysis (IOTA) Group. Terms, definitions and measurements to describe the sonographic features of adnexal tumors: a consensus opinion from the International Ovarian Tumor Analysis (IOTA) Group. Ultrasound Obstet Gynecol. 2000; 16(5): 500-505, doi: 10.1046/j.1469-0705.2000.0 0287.x, indexed in Pubmed: 11169340.

21. Timmerman D, Van Calster B, Testa A, et al. Predicting the risk of malignancy in adnexal masses based on the Simple Rules from the International Ovarian Tumor Analysis group. Am J Obstet Gynecol. 2016; 214(4): 424-437, doi: 10.1016/j.ajog.2016.01.007, indexed in Pubmed: 26800772

22. Timmerman D, Ameye L, Fischerova $D$, et al. Simple ultrasound rules to distinguish between benign and malignant adnexal masses before surgery: prospective validation by IOTA group. BMJ. 2010; 341: c6839, doi: 10.1136/bmj.c6839, indexed in Pubmed: 21156740. 